Tack F.M.G., Verloo M.G., 2001. Guidelines for sampling in Flanders (Belgium). The Science of the Total Environment 264 (1-2), 187-191.

The original publication is available at http://www.elsevier.com/

\title{
Guidelines for sampling in Flanders (Belgium)
}

\author{
F.M.G. Tack, M.G. Verloo \\ Laboratorium voor Analytische Chemie en Toegepaste Ecochemie, Universiteit Gent, \\ Coupure Links 653, B-9000 Gent, Belgium
}

\begin{abstract}
In Flanders, sampling guidelines for investigations on contamination of soils are in development. One detailed sampling guideline is currently available with respect to the prospective investigation in the frame of the Decree related to soil sanitation. Four strategies for sampling may be followed as a function of available information about pollution sources. Guidelines indicate what has to be sampled (top soil, deeper soil, soil water), how sampling points must be determined, how the number of samples and analyses is determined and what parameters need to be analysed. The example given shows that sampling guidelines will strongly depend on the ultimate goal of a particular sampling. A sampling procedure should be developed with close consideration of practical aspects and limitations, in order to be practical, realistic and cost-effective.
\end{abstract}

Keywords: sampling; soil pollution; standardisation

\section{Introduction}

The importance of sampling and sample preparation for the quality of any analysis is currently being recognised. There is a need for guidelines on good sampling practices. An international standardisation and harmonisation of sampling approaches would benefit the comparability of soil pollution studies between countries. The CEEM-Soil project is an important initiative in this context and includes the study of the soil sampling strategies and guidelines in Europe as one of its goals (Wagner et al., this issue). In this contribution, the current status of standardisation of sampling procedures in Flanders is reviewed. At the time of writing, one sampling guideline was effectively available and is discussed in some detail. It is attempted to highlight experiences that may contribute in drawing the concept for a harmonisation at the European level.

\section{History and current developments}

In recent years, Belgium has evolved into a federal structure (http://belgium.fgov.be/). It is a Federal State which consists of three communities and three regions. The federal State 
retains important areas of competence including: foreign affairs, defence, justice, finances, social security, important sectors of public health and domestic affairs, etc. The Communities and Regions are entitled to run foreign relations themselves in those areas where they have competence. Environment belongs to the authority of the regions. As a consequence, guidelines related to the investigation of metal pollution are developed at the regional level.

The regions are the Flemish Region, the Brussels-Capital Region and the Walloon Region. Developments in the field of the investigation of soil pollution are very recent in all regions. In Flanders at the time of writing, one sampling guideline has been published, which relates to the so-called prospective investigation in the frame of the decree related to soil sanitation. No such elaborated guidelines are published yet in the other regions.

The Public Waste Agency of Flanders (OVAM) published a manual for the analysis of waste materials in 1992 (Afvalstoffenanalyse compendium, 1996). That manual handles aspects of sampling and sample conservation, but does not deal with sampling strategies. On 22 February 1995, the decree related to soil sanitation (Bodemsaneringsdecreet) was issued. The practical implementation of that decree was elaborated in VLAREBO, published approximately one year later on 5 March 1996. It outlines the general procedure for investigating contaminated soils. Investigation of a potentially polluted site must start with a prospective investigation (oriënterend bodemonderzoek). When presence of pollution is shown, a descriptive investigation (beschrijvend bodemonderzoek) must be undertaken.

Details of how the prospective investigation should be conducted were published in January 1997 by the Public Waste Agency of Flanders (Oriënterend bodemonderzoek. Standaardprocedure, 1997). This document constitutes the first complete and detailed official sampling guideline available in Belgium. It includes detailed guidelines for sampling strategies to be followed in the frame of the prospective investigation. For matters related to sampling technique, sample conservation and chemical analysis, reference is made to the Afvalstoffenanalysencompendium (Afvalstoffenanalyse compendium, 1996). Guidelines for the descriptive investigation are currently in preparation.

Soil sampling guidelines are not actually incorporated in the law. The legislation merely provides soil quality standards and a procedural frame for carrying out soil sanitation studies. Detailed soil sampling guidelines including technical aspects are filled in by the Public Waste Agency of Flanders (OVAM), which as controlling body has the power to impose these guidelines and to require that these are followed.

\section{The standard procedure for the prospective investigation}

The goal of the prospective soil investigation as defined in the decree related to soil sanitation is to determine whether there are serious indications for the presence of soil pollution in the investigated soil. It should result in a description of the degree of soil pollution with reference to the available standards for soil quality. Prospective investigation should yield a conclusion with respect to the pollution status of a cadastral parcel. Only experts in soil sanitation recognised by OVAM are allowed to perform investigations in the frame of the decree related to soil pollution. For recognition, experts must submit their application to OVAM. Among the criteria for eligibility are the scientific education and years of field experience .

Previous to sampling, information in relation to administrative aspects, history, and geological and hydrological features of the site must be collected in a preliminary investigation step. Based on this information, one of five sampling strategies is selected. 
Sampling strategy 0 is to be followed when a soil sanitation that is non-conform to the decree has been performed in the past. The procedure that must be followed is elaborated in consultation with OVAM.

- Sampling strategy 1 is applied when either an prospective investigation or a mandatory soil sanitation has already been performed in the past on the parcel concerned. Based on the preliminary investigation, selected pollutants can be suspected to have caused pollution and potentially polluted spots are known. Investigation may be focussed only on these pollutants and suspected spots.

- Sampling strategy 2 must be followed when the preliminary investigation revealed that potential pollution sources may have caused a homogeneously spread pollution.

- Sampling strategy 3 is applied when the preliminary investigation revealed that a possible pollution source resulted in a heterogeneously spread pollution. To apply sampling strategy 3 , one must be able to locate potentially polluted spots based on the information obtained during the preliminary investigation.

- Sampling strategy 4 is followed when no information about potential sources of pollution is available, i.e., when nothing is known about potential pollution.

For each of the sampling strategies, the guidelines indicate what has to be sampled (top soil, deeper soil, soil water), how sampling points must be determined, how the number of samples and analyses is determined and what parameters need to be analysed. They are presented as a framework guiding the decisions of the investigator, rather than as a strict set of rules. This will probably be a prerequisite for any standardisation effort to be successful and operational. Because sampling conditions may be very site specific, there will always remain a need for an expertise to decide upon the most appropriate approach in the context of the particular site-specific circumstances.

The distinction between the different sampling strategies serves the practical purpose of minimising needed efforts and costs. Sampling is adapted to the particular site, based on information obtained from the preliminary investigation. As such, a maximum efficiency in sampling and analysis may be achieved. A sampling guideline should indeed incorporate such practical considerations in order to be realistic, feasible and operational.

\section{The example of sampling strategy 2}

To illustrate the concept of the sampling guidelines provided with the standard procedure for the prospective investigation, the example of Pollution Strategy 2 will be given. This is the strategy prescribed for a case where a pollution source resulted in a homogeneously distributed pollution.

\subsection{What to sample}

Guidelines are provided to let the expert decide what has to be sampled. As mentioned before, only a decision framework is given as a function of the appropriate sampling strategy. According to the guidelines, borings should be performed to such a depth 'that the suspected layer and immediately adjacent layers can be sampled'. If pollution is expected to be in the upper soil, sampling should be done over a depth between 0.5 and 2 meter. This depth is to be determined - and argued - by the investigator as a function of the characteristics of the polluting substance and soil properties. For substances that are not expected to be a cause of 
pollution in the site under investigation, sampling should be performed to at least $0.5 \mathrm{~m}$ depth in the undisturbed soil profile.

Any guideline should be written to be as clear and unambiguous possible. The currently discussed guideline could be somewhat improved in that respect. For example, sampling depth is specified in case the suspected substance is present in the upper soil layer. Currently, the document does not explicitly indicate how the approach should be if pollution is expected to occur in deeper layers. At the end of the paragraph, it is given what the minimum requirements of depth are for unsuspected substances, i.e., substances that are always to be analysed, but that are not suspected to be present in the currently investigated soil. There it is mentioned that the depth should minimally be $0.5 \mathrm{~m}$ in the 'original, undisturbed soil profile'. Should we therefore understand that the previously given depth between 0.5 and $2 \mathrm{~m}$ also refers to the 'original, undisturbed soil profile'? Phrasing and correct wording is very important to minimise any confusion or uncertainty that may arise during implementation of the soil sampling guidelines.

\subsection{Sampling strategy}

The sampling strategy is vaguely defined. It is specified that 'borings are systematically distributed over the parcel'. Although the guidelines explicitly start from the premise that experts perform the sampling, procedures for selecting the sampling points could be more specific. Two methods for sampling a small area of up to 3 or 4 hectare are commonly recommended (Rubio and Ure, 1993). In the first, samples are taken along a W shaped track. In the second, samples are taken along a zig-zag track, taking one sampling from each sampling unit. The degree of standardisation could be improved by specifying one of such methods. This would ensure that variability that may be related to the use of different sampling strategies is eliminated.

In contrast, the number of sampling points is clearly defined. Required numbers are concisely given as a function of surface area (Table 1). The number of samples required depends on the scientific objectives, and may also be constrained by cost and time factors (Ramsey, 1997). Indicating concise requirements with respect to the number of samples therefore is only sensible and possible if the target of the sampling is well defined. Any standardisation and harmonisation effort of sampling approaches should indeed start with defining the context and target of the sampling.

\subsection{Parameters to analyse and number of samples that has to be analysed}

As a practical measure to reduce the efforts and costs, not all sampled points must be analysed for all parameters. Some samples must be analysed for both the suspected pollutants and standard set of parameters (SAP), other samples need analysis for SAP only (Table 2). The required numbers are unambiguously given as a function of the sampled surface area (Table 2). Selection of these samples that are to be analysed may be based on sensory perception, if possible. It is anyhow left to the decision of the expert.

\section{Conclusions}

A harmonisation of technical aspects with respect to soil sampling is on its way in Flanders. Currently, sampling guidelines for one specific purpose, i.e., in the frame of the prospective investigation to detect and identify polluted soils, are available. A number of principles applied in the Flemish approach are valuable and need consideration when harmonisation of sampling procedures will be developed at the European level. 
The Flemish harmonisation of sampling procedures starts from the premise that experts will guide the sampling. Sampling guidelines therefore are conceived as a framework within which decisions are left to the expert rather than as a rigid set of rules. This build-in degree of freedom in sampling guidelines will probably be required in order to cope with the variability of different sites in a realistic way. Obviously, the expert should always be required to justify why he adopted a particular approach.

Any protocol for sampling will very much be determined by the ultimate purpose of the sampling. As a result, standardisation efforts will imply the development of different sampling protocols as a function of various objectives. It does not make sense to compare different sampling strategies if they are not aimed at exactly the same goal, i.e., if they have not been designed to obtain the same type of information at the same level of detail.

A sampling procedure should be developed with close consideration of practical aspects and limitations in order to be practical, realistic and cost-effective. One sampling guideline corresponds with one specific target, but within that target, further reduction of costs and efforts may be obtained by distinguishing typical cases.

A guideline should be written as clearly and concisely as possible to avoid misinterpretations, ambiguities or confusions. Although freedom should be left to the investigator, the limits of that freedom must be clearly delineated.

Sampling guidelines are an urgent need in order to facilitate interpretation and comparability of our data. Harmonising standard procedures for sampling may prove even more diverse and complicated than is the exercise of standardising analytical procedures. We hope that current expertise within the European countries may contribute to this difficult task.

\section{References}

Afvalstoffenanalyse compendium. Openbare Afvalstoffenmaatschappij voor het Vlaamse Gewest, Mechelen, België, 1996.

Oriënterend bodemonderzoek. Standaardprocedure. Openbare Afvalstoffenmaatschappij voor het Vlaamse Gewest, Mechelen, België, 1997, 44 pp.

Ramsey MH. Sampling and sample preparation. In: Gill R, editor. Modern analytical geochemistry. Addison Wesley Longman Limited, Essex, England, 1997, pp. 12-28.

Rubio R, Ure AM. Approaches to sampling and sample pretreatments for metal speciation in soils and sediments. Int J Environ Anal Chem 1993; 51: 205-217.

Wagner G, Mohr ME, Sprengart J, Desaules A, Muntau H, Theocharopoulos S, Quevauviller P. Objectives, concept and design of the CEEM Soil project. Sci Total Environ this issue; 
Table 1. Guidelines for determining the number of sampling points and samples to be analysed for sampling strategy 2, assuming homogeneously distributed pollution (Oriënterend bodemonderzoek. Standaardprocedure, 1997)

\begin{tabular}{ccccc}
\hline $\begin{array}{c}\text { Surface area } \\
\text { (ha) }\end{array}$ & $\begin{array}{c}\text { Number of } \\
\text { borings }\end{array}$ & \multicolumn{3}{c}{ Number of samples to be analysed } \\
\cline { 3 - 5 } & & total & $\begin{array}{c}\text { analysis of SAP } \\
\text { and suspected } \\
\text { substance }\end{array}$ & analysis of SAP \\
\hline$<0.2$ & 4 & 3 & 2 & 1 \\
$0.2-1$ & 5 & 3 & 2 & 2 \\
$1-2$ & 6 & 4 & 2 & 2 \\
$2-3$ & 7 & 5 & 3 & 3 \\
$3-4$ & 8 & 6 & 4 & 3 \\
$4-5$ & 9 & 7 & 4 & 2 \\
$5-6$ & 10 & 8 & 5 & \\
\hline
\end{tabular}

${ }^{1}$ SAP: standard set of parameters 
Table 2. Standard parameters for analysis in the frame of a prospective investigation (Oriënterend bodemonderzoek. Standaardprocedure, 1997)

\begin{tabular}{lcc}
\hline Parameters to analyse & Analyses for solid part of soil & Analyses for soil water \\
\hline $\mathrm{pH}$ & $+(1)$ & + \\
Electrical conductivity and & - & + \\
temperature & + & \\
Dry matter content & $+(1)$ & \\
Organic matter content & $+(1)$ & - \\
Clay content & + & $+(2)$ \\
Heavy metals $(\mathrm{Pb}, \mathrm{Zn}, \mathrm{Cd}$, & & + \\
$\mathrm{Cu}, \mathrm{Ni}, \mathrm{As}, \mathrm{Hg}, \mathrm{Cr})$ & - & + \\
$\mathrm{BTEX}$ & + & - \\
Mineral oils & $+(2)$ & \\
Polycyclic aromatic & & \\
hydrocarbons $(10 \mathrm{PAH})$ & $+(2)$ & $+(2)$ \\
EOX & - & \\
VOCl & - & \\
\hline
\end{tabular}

PAH: naphthalene, benzo(a)pyrene, fenanthrene, fluoranthene, benzo(a)anthracene, chrysene, benzo(b)fluroanthene, benzo(k)fluoranthene, Benzo(ghi)perylene and indeno(1,2,3-cd)pyrene BTEX: benzene, toluene, ethylbenzene and xylene

EOX: Extractable chlorinated hydrocarbons

VOCl: Volatile chlorinated hydrocarbons

(1) Not all samples must be analysed for these parameters. For $\mathrm{pH}$, clay content and organic matter content, a few samples that are representative for the parcel are sufficient.

(2) For these parameters, only half of the required number of samples is to be analysed. 